\title{
Synthesis of Gold(III) Complexes with Bidentate Amino-Thiolate Ligands as Precursors of Novel Bifunctional Acyclic Diaminocarbenes
}

\author{
Sara Montanel-Pérez, Anabel Izaga, Antonio Laguna, M. Dolores Villacampa,* \\ and M. Concepción Gimeno*(1)
} Departamento de Química Inorgánica, Instituto de Síntesis Química y Catálisis Homogénea (ISQCH), CSIC-Universidad de
Zaragoza, E-50009 Zaragoza, Spain

\section{Supporting Information}

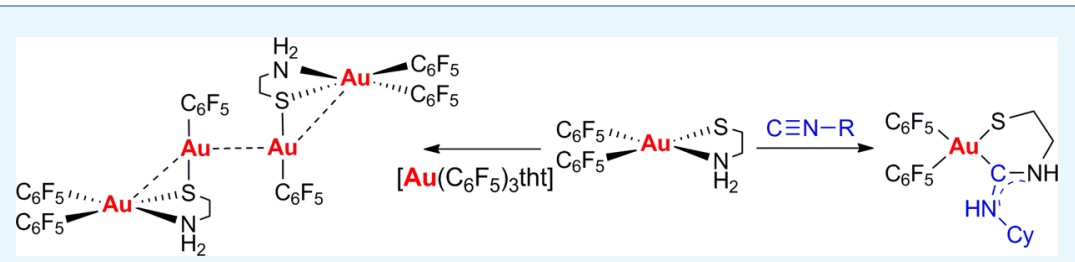

ABSTRACT: Two neutral bis(pentafluorophenyl)thiolate gold(III) complexes with the unsymmetrical $S^{\wedge} \mathrm{N}$ ligands 2 aminothiophenol or cysteamine have been synthesized and their reactivity has been studied. Homo- and heterodinuclear compounds were obtained by their coordination to gold(I) or silver(I) derivatives through the sulfur atom. Interestingly, a

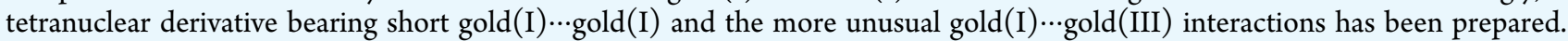
These amino-thiolate derivatives can be used as precursors for the synthesis of novel gold(III) acyclic diaminocarbene complexes by reaction with isocyanides CNR. The nucleophilic attack of the amino group to isocyanide molecules affords the synthesis of unprecedented bidentate $C^{\wedge} S$ acyclic diaminocarbene ligands. All of the complexes are air- and moisture-stable at room temperature and have been spectroscopically and structurally characterized.

\section{INTRODUCTION}

The chemistry of gold has undergone a big development in the last decades mainly because of the great range of applications presented by its complexes. However, this is really true for gold(I) derivatives. The chemistry of gold(III) is an active area but has been less explored than gold(I) chemistry probably because of the instability of gold(III) species to reduction. ${ }^{1}$ Consequently, the synthesis of gold(III) derivatives has been the subject of increasing interest mainly because of its versatile applications as catalysts, ${ }^{2}$ as luminescent materials, ${ }^{3}$ or as antitumor or antiviral agents. ${ }^{4}$ Gold trichloride was one of the first gold catalyst used, and from then on, several gold(III) complexes bearing bidentate ligands have been reported as excellent catalysts in some organic transformations. ${ }^{5}$ In addition, excellent photophysical properties have been found in tridentate cyclometallated gold(III) complexes bearing ancillary ligands such as $N$-heterocyclic carbenes, alkynyls, amides, or thiolates. ${ }^{6}$ Biological studies of gold(III) derivatives have been developed in part because they are isostructural and isoelectronic to platinum(II) complexes. It was supposedly a cytotoxic activity similar to that of cisplatin, the most wide metal-based antitumor drug. However, in physiological conditions, gold(III) can be reduced to gold(I). For this reason, the election of suitable ligands to stabilize the gold(III) complexes to prevent their reduction is important. For example, metallacyclic gold(III) complexes ${ }^{7}$ or chelated gold(III) derivatives ${ }^{7}$ with multidentate ligands have been stablished as far more stable under physiological conditions than any other, and some of them have been identified as good antitumor agents. ${ }^{7}$

Gold(III) thiolate derivatives are less represented than the related gold(I) species probably because of the ability of thiolate groups to reduce gold(III) to gold(I) ${ }^{8}$ although, for example, some tetrathiolate gold(III) compounds with highly electron-deficient thiols (highly fluorinated), that may be less capable of reducing gold(III), were synthesized by Bachman et al., and some others with pentafluorophenyl groups have been described. ${ }^{10}$ Moreover, an important number of metallacyclic gold(III) complexes with thiolate ligands have been reported, and some of them have been identified as good antitumor agents. $^{11}$

In this work, we were aiming at preparing stable gold(III) derivatives bearing bidentate $S^{\wedge} \mathrm{N}$ and $\mathrm{S}^{\wedge} \mathrm{C}$ ligands. Mono- and binuclear thiolate gold(III) derivatives with potential medicinal properties have been prepared. Two heterofunctional ligands, 2 -aminothiophenol and cysteamine, have been used to synthesize the corresponding bis(pentafluorophenyl)(thiolate)gold(III) derivatives. We have chosen both ligands because of their different functional groups, the thiolate sulfur atom, that provide stability to the compound in addition to

Received: July 5, 2018

Accepted: September 26, 2018

Published: October 11, 2018 
provide further reactivity by coordination of other metals, and the amino group that is a reactive group can be further functionalized. Thus, the bis(pentafluorophenyl)(thiolate)gold(III) complexes can act as metalloligands through the donor sulfur atom to give homo- and hetero-bimetallic complexes. Moreover, the amine nitrogen $\left(\mathrm{NH}_{2}\right)$ of the ligands can react with isocyanide groups to give the corresponding gold(III) derivatives with unprecedented bidentate $S^{\wedge} C$ acyclic diaminocarbenes. These ligands, similar to the widely studied N-heterocyclic carbenes, are being the subject of a great attention because of their excellent possibilities to modulate both steric and electronic properties, as well as the easier mode to obtain chiral derivatives compared to the $N$-heterocyclic carbenes. ${ }^{12}$ The reactivity of gold-amine complexes with isocyanide molecules or gold isocyanide compounds with amines to give the corresponding acyclic diaminocarbene complexes has been stablished for gold(I) and gold(III) derivatives. ${ }^{13}$ Most of the acyclic diaminocarbenes described are mono (Figure $1 \mathrm{a})^{13 \mathrm{~b}, \mathrm{c}}$ or dicarbene (Figure
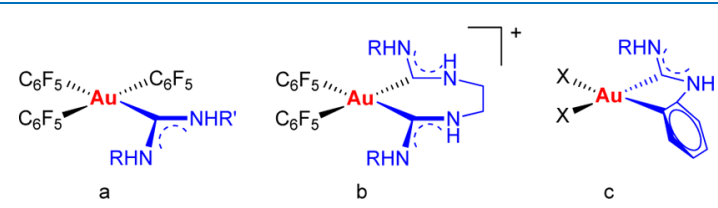

Figure 1. Gold(III) acyclic diaminocarbenes.

1b) ${ }^{13 d}$ gold(III) species, but we have previously reported functionalized cyclic diaminocarbenes, originating from the reaction of cyclometalled complexes containing an amine group with isocyanides (Figure 1c). ${ }^{13 g}$

Following this strategy of using bidentate heterofunctional amine ligands bonded to a gold(III) center, we have chosen aminothiolates because they underwent a reaction with isocyanide ligands in the gold coordination sphere, without reduction or decomposition, allowing the synthesis of a novel bidentate-chelated $\mathrm{C}^{\wedge} \mathrm{S}$ acyclic aminocarbene ligands.

\section{RESULTS AND DISCUSSION}

The gold(III) complexes have been prepared starting from the cis- $\left[\mathrm{Au}\left(\mathrm{C}_{6} \mathrm{~F}_{5}\right)_{2}\left(\mathrm{OEt}_{2}\right)_{2}\right] \mathrm{ClO}_{4}$ precursor which has two labile diethyl ether molecules. In this work, two functionalized amines, 2-aminothiophenol and cysteamine, were used to prepare the uncharged starting materials 1 and 2, respectively, (Scheme 1) as yellow solids that are air- and moisture-stable solids. These derivatives, as well as the following derivatives, have been characterized by means of infrared (IR), elemental analysis, NMR spectroscopy, and mass spectrometry. Assignments of the ${ }^{1} \mathrm{H}$ NMR and ${ }^{13} \mathrm{C}$ NMR signals were made on the

Scheme 1. Synthesis of Complexes 1 and 2

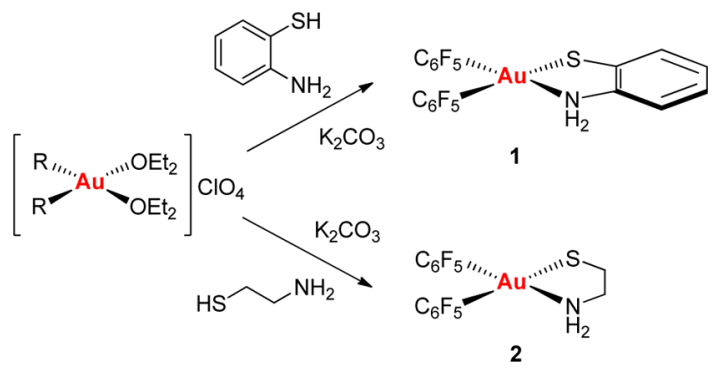

basis of two-dimensional (2D) correlation spectroscopy and heteronuclear single-quantum correlation spectra.

The IR spectra of $\mathbf{1}$ and $\mathbf{2}$ present, apart from other absorptions, the vibrations because of the pentafluorophenyl groups bonded to gold(III) at 1503 (s), $952(\mathrm{~s}), 801$ (s), and $792(\mathrm{~s}) \mathrm{cm}^{-1}$ for 1 or $1507(\mathrm{~s}), 960(\mathrm{~s}), 811(\mathrm{~m})$, and $797(\mathrm{~m})$ $\mathrm{cm}^{-1}$ for $2 .{ }^{1} \mathrm{H}$ NMR spectra show the expected resonances for the ligands with a different chemical shift. The ${ }^{19} \mathrm{~F}$ NMR spectrum of each compound presents six resonances, two for the para fluorines consistent with two different $\mathrm{C}_{6} \mathrm{~F}_{5}$ and two for both the ortho and the meta fluorines. This indicates that the pentafluorophenyl rings can rotate around the goldcarbon bond at room temperature. In the $\mathrm{ESI}^{+}$mass spectra, the fragments $[\mathrm{M}+\mathrm{H}]^{+}$appear at $m / z=656(15 \%)(1)$ and 608 (76\%) (2).

Complexes $\mathbf{1}$ and $\mathbf{2}$ were used as metalloligands through the electronically rich sulfur atom, which has lone electron pairs that can be donated to metal cations. Then, the treatment of 1 with equimolecular amounts of $\left[\mathrm{Au}(\mathrm{OTf})\left(\mathrm{PPh}_{3}\right)\right]$ or $\mathrm{AgOTf}$ gave the bimetallic $\mathrm{Au}(\mathrm{III})-\mathrm{Au}(\mathrm{I})$ (3) or $\mathrm{Au}(\mathrm{III})-\mathrm{Ag}(\mathrm{I})$ (4) complexes as yellow or white, respectively, air- and moisturestable solids (Scheme 2).

\section{Scheme 2. Synthesis of Complexes 3 and 4}

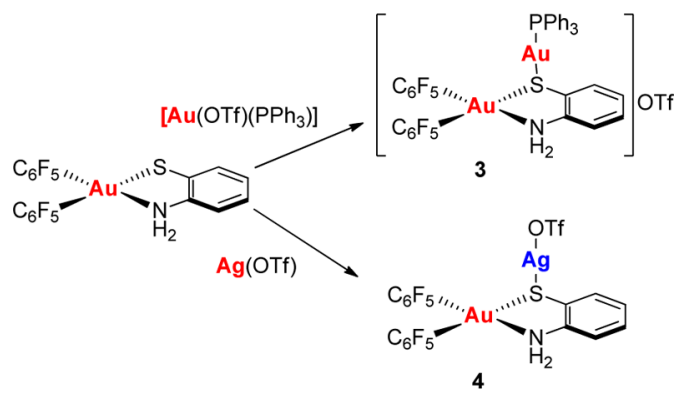

The IR spectra of $\mathbf{3}$ and $\mathbf{4}$ show the absorption for the anion trifluoromethanesulphonate around 1265, 1230, 1170, and $1023 \mathrm{~cm}^{-1}$. The typical absorption of the pentafluorophenyl groups appear around $1500(\mathrm{~s}), 965(\mathrm{~s}), 813(\mathrm{~m})$, and 800 (m) $\mathrm{cm}^{-1}$. The ${ }^{1} \mathrm{H}$ NMR spectrum of 3 displays resonances for the 2-aminothiophenol ligand and phenyl rings in the appropriate ratio. The ${ }^{31} \mathrm{P}\left\{{ }^{1} \mathrm{H}\right\}$ NMR spectrum of 3 shows a single resonance for the phosphorus of the triphenylphosphine group. Two different pentafluorophenyl moieties, with free rotation in the ring, can be identified in the ${ }^{19} \mathrm{~F}$ NMR spectra of 3 and 4.

The reaction of complex 2 with the gold(III) derivative $\left[\mathrm{Au}\left(\mathrm{C}_{6} \mathrm{~F}_{5}\right)_{3}(\mathrm{tht})\right]$ (tht = tetrahydrothiophene) afforded the unexpected binuclear gold(III)-gold(I) complex 5 as a yellow air- and moisture-stable solid (Scheme 3 ). The gold(III) of the starting material, $\left[\mathrm{Au}\left(\mathrm{C}_{6} \mathrm{~F}_{5}\right)_{3}(\mathrm{tht})\right]$, has been reduced to gold(I), and the oxidation of pentafluorophenyl with the

\section{Scheme 3. Synthesis of Complex 5}

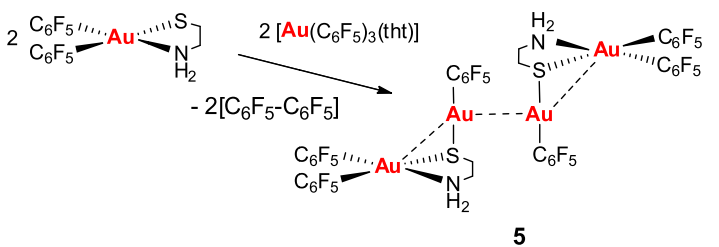


formation of decafluorobiphenyl has taken place. The latter compound has been easily identified in the ${ }^{19} \mathrm{~F}$ NMR spectrum of complex 5. Additionally, this compound can be prepared by reaction of the starting complex 2 with $\left[\mathrm{Au}\left(\mathrm{C}_{6} \mathrm{~F}_{5}\right)(\mathrm{tht})\right]$.

The IR spectrum of complex 5 shows the absorptions of pentafluorophenyl groups bonded to gold(I) at 1485, 948, and $784 \mathrm{~cm}^{-1}$ and those bonded to $\mathrm{Au}$ (III) at 1505, 959, 815, and $794 \mathrm{~cm}^{-1}$. The ${ }^{1} \mathrm{H}$ NMR spectrum shows the expected resonances for the ligand with a different chemical shift. In the ${ }^{19} \mathrm{~F}$ NMR spectrum, the three signals corresponding to the gold(I) fragment and the six signals corresponding to the gold(III) fragment were observed in an appropriate ratio.

The crystal structure of complex $\mathbf{5}$ has been determined by an X-ray diffraction study (Figure 2). Two different crystals in

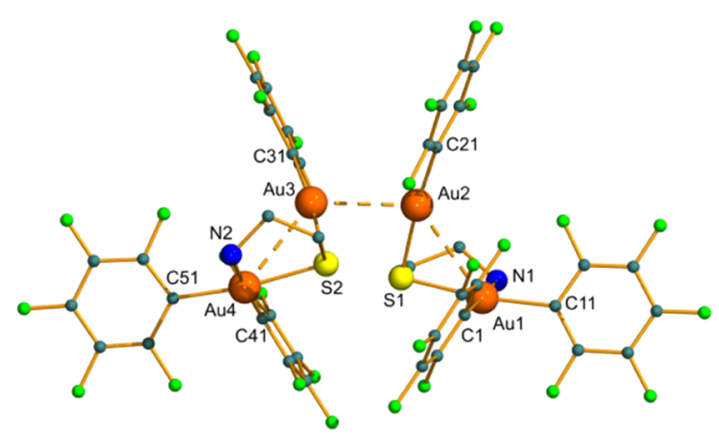

Figure 2. Molecular structure of complex 5. Hydrogen atoms are omitted for clarity. Selected bond lengths $(\AA)$ and angles $\left({ }^{\circ}\right): \mathrm{Au}(1)-$ $\mathrm{C}(1)$ 2.012(6), $\mathrm{Au}(1)-\mathrm{C}(11) 2.049(7), \mathrm{Au}(1)-\mathrm{N}(1)$ 2.089(6), $\mathrm{Au}(1)-\mathrm{S}(1)$ 2.344(2), $\mathrm{Au}(1)-\mathrm{Au}(2)$ 3.175(1), $\mathrm{Au}(2)-\mathrm{C}(21)$ 2.011(7), $\mathrm{Au}(2)-\mathrm{S}(1)$ 2.340(2), $\mathrm{Au}(2)-\mathrm{Au}(3)$ 3.054(1), $\mathrm{Au}(3)-$ $\mathrm{C}(31)$ 2.031(6), $\mathrm{Au}(3)-\mathrm{S}(2)$ 2.331(2), $\mathrm{Au}(3)-\mathrm{Au}(4)$ 3.214(1), $\mathrm{Au}(4)-\mathrm{C}(41)$ 2.011(7), $\mathrm{Au}(4)-\mathrm{C}(51)$ 2.019(7), $\mathrm{Au}(4)-\mathrm{N}(2)$ 2.101(6), $\mathrm{Au}(4)-\mathrm{S}(2)$ 2.345(2), $\mathrm{C}(1)-\mathrm{Au}(1)-\mathrm{C}(11)$ 92.0(3), $\mathrm{C}(11)-\mathrm{Au}(1)-\mathrm{N}(1)$ 91.1(3), C(1)-Au(1)-S(1) 90.5(2), N(1)$\mathrm{Au}(1)-\mathrm{S}(1)$ 86.5(2), C(21)-Au(2)-S(1) 173.4(2), C(31)-Au(3)$\mathrm{S}(2)$ 173.8(2), C(41)-Au(4)-C(51) 91.9(3), C(51)-Au(4)-N(2) 92.0(3), C(41)-Au(4)-S(2) 89.6(2), N(2)-Au(4)-S(2) 86.6(2).

a 95.5:4.5 ratio were identified in compound 5. Only four gold atoms of the minority crystal could be detected. Several attempts to crystallize a single crystal were unfruitful; in all of the cases, a similar twinning was observed. The complex crystallizes in the triclinic $P \overline{1}$ space group as a tetramer formed by an intermolecular gold(I)-gold(I) interaction of 3.054(1) Å. Moreover, there are short intramolecular gold(I)-gold(III) distances of 3.175(1) and 3.214(1) $\AA$ which indicate a high degree of metal-metal interaction. These distances are shorter than those found in other multinuclear gold(I)-gold(III) complexes, for example, the tetranuclear complex $\left[\left(\mathrm{C}_{6} \mathrm{~F}_{5}\right)_{3} \mathrm{Au}-\right.$ $\left.\left(\mu_{2}-2-\mathrm{SC}_{6} \mathrm{H}_{4} \mathrm{NH}_{2}\right)(\mathrm{AudppmAu})\left(\mu_{2}-2-\mathrm{SC}_{6} \mathrm{H}_{4} \mathrm{NH}_{2}\right) \mathrm{Au}\left(\mathrm{C}_{6} \mathrm{~F}_{5}\right)_{3}\right]$ (dppm $=\mathrm{PPh}_{2} \mathrm{CH}_{2} \mathrm{PPh}_{2}$ ) with the 2-aminothiophenol ligand $[\mathrm{Au}(\mathrm{III})-\mathrm{Au}(\mathrm{I})$ from $3.2812(7)$ to $3.4052(7) \AA],{ }^{8 \mathrm{f}}$ or with the bridging sulfido ligands in $\left[\left(\mathrm{C}_{6} \mathrm{~F}_{5}\right)_{2} \mathrm{Au}\left\{\mu-\left(\mathrm{S}\left(\mathrm{Au}_{2} \mathrm{dppf}\right)\right\}_{2}\right] \mathrm{OTf}\right.$ for which the shortest distance found is $3.2195(8) \AA^{14}$ Ignoring metal-metal interactions, the gold atoms show in 5 the characteristic geometry of each oxidation number, that is, square planar for gold(III) and linear for gold(I) atoms.

The reactivity of gold-amine complexes with isocyanide molecules to give the corresponding acyclic diaminocarbene complexes has been established for amine gold(I) and gold(III) derivatives. ${ }^{13}$ In this work, the reaction of $\mathbf{1}$ or 2 with an equimolecular amount of the corresponding isocyanide
CNR ( $\mathrm{R}=$ cyclohexyl, 2-naphthyl) gives complexes 6-8 in which the nucleophilic attack of the amine to the isocyanide carbon produces the formation of unprecedented bidentate $\mathrm{S}^{\wedge} \mathrm{C}$ acyclic diaminocarbenes (Scheme 4). Compounds 6-8

Scheme 4. Synthesis of Complexes 6-8

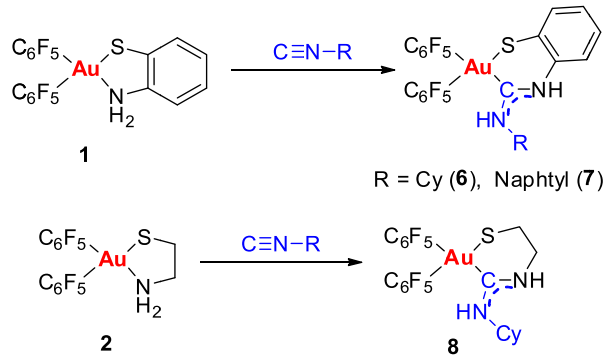

are synthesized as off-white air- and moisture-stable solids. Their ${ }^{1} \mathrm{H}$ NMR spectra show the resonances assigned to the protons of the thiolate ligands and the signals characteristic of the substituents of the former isocyanide group in the appropriate ratio. The ${ }^{19} \mathrm{~F}$ NMR spectra of complexes indicate that the pentafluorophenyl rings can rotate at room temperature. In the $\mathrm{ESI}^{+}$mass spectra, the fragments $\left[\mathrm{M}+\mathrm{Na}^{+}(6)\right.$ and $[\mathrm{M}+\mathrm{H}]^{+}(8)$ appear at $m / z(\%)=787(51)$ and 717 (100), respectively.

The structure of complex $\mathbf{8}$ in the solid state has been solved by an X-ray diffraction study (Figure 3 ). It crystallizes in the

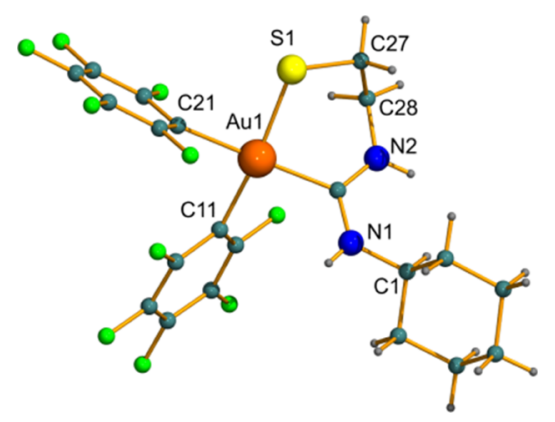

Figure 3. Diagram of complex 8. Selected bond lengths $[\AA]$ and angles $\left[^{\circ}\right]$ for complex 8: $\mathrm{Au}(1)-\mathrm{C}(11)$ 2.061(5); $\mathrm{Au}(1)-\mathrm{C}(21)$ 2.073(5); $\mathrm{Au}(1)-\mathrm{C}(29)$ 2.052(6); $\mathrm{Au}(1)-\mathrm{S}(1) 2.331(1) ; \mathrm{N}(1)-$ $\mathrm{C}(29) \quad 1.324(7) ; \mathrm{N}(2)-\mathrm{C}(29) \quad 1.313(7) ; \mathrm{C}(11)-\mathrm{Au}(1)-\mathrm{C}(29)$ 91.0(2); $\mathrm{C}(11)-\mathrm{Au}(1)-\mathrm{C}(21) \quad 91.8(2) ; \quad \mathrm{C}(29)-\mathrm{Au}(1)-\mathrm{S}(1)$ 93.4(2); $\mathrm{C}(21)-\mathrm{Au}(1)-\mathrm{S}(1)$ 84.4(1).

monoclinic $P 2(1) / n$ space group with one molecule in the asymmetric unit. The gold(III) center is in a distorted squareplanar geometry. The mean deviation from the plane formed by the four donor atoms of the gold center is $0.0475 \AA$ (S1, $\mathrm{C} 29, \mathrm{C} 11$, and C21). The angles around the gold(III) atom range from $84.40(16)^{\circ}$ to $93.39(15)^{\circ}$. The six-membered $\mathrm{AuC}_{3} \mathrm{SN}$ chelate ring adopts a boat conformation, as shown in Figure 3, with the Au atom and the methylene carbon lying on the same side of the plane of the other four atoms, $\mathrm{C}_{2} \mathrm{SN}$, which are almost coplanar (similar boat conformations were observed for some of us in the bidentate $\mathrm{C}^{\wedge} \mathrm{N}$ gold(III) acyclic carbene derivatives reported in ref $13 \mathrm{~g}$ ).

\section{CONCLUSIONS}

In summary, new gold(III) complexes with aminothiolate ligands have been synthesized. These compounds are very 
stable and have allowed the preparation of polynuclear species by reaction with gold(I) or silver(I) moieties, in which these metals coordinate to the thiolate sulfur atom. Interestingly, the reaction with other gold(III) centers in the form of a tris(pentafluorophenyl) derivative produces reduction to gold(I) and oxidation of the two pentafluorophenyl units to form decafluorobiphenyl. In this case, it is probable that bulky aryl groups preclude the formation of the corresponding thiolate ligand bridging to bulky gold(III) fragments. An interesting tetranuclear species is formed which shows the

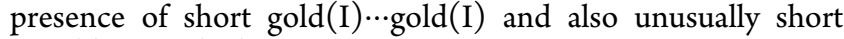
gold(I) $\cdots$ gold(III) interaction contacts. In addition, these heterofunctional amine-thiolate complexes serve as excellent platforms to form unprecedented $S^{\wedge} \mathrm{C}$ bidentate acyclic diaminocarbenes by reaction with isocyanides. These complexes are very stable and may be adequate for biological studies.

\section{EXPERIMENTAL SECTION}

Instrumentation. $\mathrm{C}, \mathrm{H}, \mathrm{N}$, and $\mathrm{S}$ analyses were carried out with a PerkinElmer 2400 microanalyzer. Mass spectra were recorded on a VG AutoSpec with the ESI technique. ${ }^{1} \mathrm{H}$, ${ }^{13} \mathrm{C}\left\{{ }^{1} \mathrm{H}\right\}$, and ${ }^{19} \mathrm{~F} \mathrm{NMR}$, including $2 \mathrm{D}$ experiments, were recorded at room temperature on a Bruker AVANCE 400 spectrometer $\left({ }^{1} \mathrm{H}, 400,{ }^{19} \mathrm{~F}, 376.5,{ }^{13} \mathrm{C}, 100.6,{ }^{31} \mathrm{P} 162 \mathrm{MHz}\right)$ and Bruker 300 spectrometer $\left({ }^{1} \mathrm{H}, 300,{ }^{19} \mathrm{~F}, 282.4,{ }^{13} \mathrm{C} 75.5\right.$, $\left.{ }^{31} \mathrm{P} 121.5 \mathrm{MHz}\right)$ with chemical shifts $(\delta, \mathrm{ppm})$ reported relative to the solvent peaks of the deuterated solvents.

Starting Materials. The starting materials [Au$\left.\left(\mathrm{C}_{6} \mathrm{~F}_{5}\right)_{2}\left(\mathrm{OEt}_{2}\right)_{2}\right] \mathrm{ClO}_{4}{ }^{15}$ and $\left[\mathrm{Au}\left(\mathrm{C}_{6} \mathrm{~F}_{5}\right)_{3}(\mathrm{tht})\right]^{16}$ were prepared by published procedures. $\left[\mathrm{Au}(\mathrm{OTf})\left(\mathrm{PPh}_{3}\right)\right]$ was obtained by the reaction of $\left[\mathrm{AuCl}\left(\mathrm{PPh}_{3}\right)\right]^{17}$ with $\mathrm{Ag}(\mathrm{OTf})$ in dichloromethane and used in situ. All other reagents were commercially available. Solvents were used as received without purification or drying.

Caution: Perchlorate salts with organic cations might be explosive.

General Procedure of the Synthesis of Complexes 1 and 2. 2-Aminothiophenol (0.0376 g, $0.3 \mathrm{mmol}, \rho=1.17 \mathrm{~g}$. $\left.\mathrm{mL}^{-1}\right)$ or cysteamine $(0.0231 \mathrm{~g}, 0.3 \mathrm{mmol})$ was added to a freshly prepared solution of $\left[\mathrm{Au}\left(\mathrm{C}_{6} \mathrm{~F}_{5}\right)_{2}\left(\mathrm{OEt}_{2}\right)_{2}\right] \mathrm{ClO}_{4}(0.2336$ $\mathrm{g}, 0.3 \mathrm{mmol})$ in diethyl ether $(20 \mathrm{~mL})$ with $\mathrm{K}_{2} \mathrm{CO}_{3}(0.0456 \mathrm{~g}$, $0.33 \mathrm{mmol}$ ). After stirring for $3 \mathrm{~h}$, the suspension was filtered over Celite. The volume was reduced to $5 \mathrm{~mL}$, and addition of $n$-hexane afforded $\mathbf{1}$ and $\mathbf{2}$ as yellow solids, which were finally filtered.

Complex 1. Yield: $0.105 \mathrm{~g}, 53 \%$. Anal. Calcd for $\mathrm{C}_{18} \mathrm{H}_{6} \mathrm{AuF}_{10} \mathrm{NS}$ (655.26): C, 32.99; H, 0.92; N, 2.14; S, 4.89. Found: C, 33.23; H, 1.14; N, 2.06; S, 4.53. IR $\left(\mathrm{cm}^{-1}\right):\left(\mathrm{NH}_{2}\right)$ : 3364; $\left(\mathrm{C}_{6} \mathrm{~F}_{5}\right):$ 1503, 952; $\left(\right.$ cis- $\left.\mathrm{C}_{6} \mathrm{~F}_{5}\right):$ 801, 792, (Au-S) 323. ${ }^{1} \mathrm{H}$ NMR $\left(\mathrm{CD}_{3}\right)_{2} \mathrm{CO}: \delta 8.13$ (br s, $\left.2 \mathrm{H}, \mathrm{NH}_{2}\right), 7.41(\mathrm{~m}, 1 \mathrm{H}, 6-$ $\mathrm{H}), 7.31\left(\mathrm{~d}, 1 \mathrm{H},{ }^{3} J_{\mathrm{H}-\mathrm{H}}=8.0 \mathrm{~Hz}, 3-\mathrm{H}\right), 7.19(\mathrm{~m}, 1 \mathrm{H}, 5-\mathrm{H})$, $7.02(\mathrm{~m}, 1 \mathrm{H}, 4-\mathrm{H})$ ppm. ${ }^{19} \mathrm{~F}$ NMR $\left(\mathrm{CD}_{3}\right)_{2} \mathrm{CO}: \delta-122.9(\mathrm{~m}$, $2 \mathrm{~F}, o-\mathrm{F}),-123.7(\mathrm{~m}, 2 \mathrm{~F}, o-\mathrm{F}),-159.9\left(\mathrm{t}, 1 \mathrm{~F}, J_{p-\mathrm{F}, m-\mathrm{F}}=19.4 \mathrm{~Hz}\right.$, $p-\mathrm{F}),-160.1\left(\mathrm{t}, 1 \mathrm{~F}, J_{p-\mathrm{F}, m-\mathrm{F}}=19.5 \mathrm{~Hz}, p-\mathrm{F}\right),-164.6(\mathrm{~m}, 2 \mathrm{~F}, m-$ $\mathrm{F}),-165.0(\mathrm{~m}, 2 \mathrm{~F}, \mathrm{~m}-\mathrm{F}) \mathrm{ppm} .{ }^{13} \mathrm{C}\left\{{ }^{1} \mathrm{H}\right\}$ NMR $\left(\mathrm{CD}_{3}\right)_{2} \mathrm{CO}: \delta$ $147.1\left(\mathrm{~m}, \mathrm{C}_{6} \mathrm{~F}_{5}\right), 144.6\left(\mathrm{~m}, \mathrm{C}_{6} \mathrm{~F}_{5}\right), 141.2,140.6$ (2s, 2C, 1-C, 2-C), $139.1\left(\mathrm{~m}, \mathrm{C}_{6} \mathrm{~F}_{5}\right), 136.6\left(\mathrm{~m}, \mathrm{C}_{6} \mathrm{~F}_{5}\right), 129.7$ (s, 1C, 6-C), 129.0 (s, 1C, 5-C), 127.3 (s, 1C, 3-C), 124.2 (s, 1C, 4-C) ppm. MS $\left(\mathrm{ESI}^{+}\right) \mathrm{m} / z(\%): 656(15)[\mathrm{M}+\mathrm{H}]^{+}$.

Complex 2. Yield: $0.140 \mathrm{~g}, 77 \%$. Anal. Calcd for $\mathrm{C}_{14} \mathrm{H}_{6} \mathrm{AuF}_{10} \mathrm{NS}$ (Pm 607.22): C, 27.69; H, 1.00; N, 2.31; S,
5.28. Found: C, 27.54; H, 1.18; N, 2.54; S, 4.89. IR ( $\left.\mathrm{cm}^{-1}\right)$ : $\left(\mathrm{NH}_{2}\right): 3329 ;\left(\mathrm{C}_{6} \mathrm{~F}_{5}\right): 1507,960 ;\left(\right.$ cis- $\left.\mathrm{C}_{6} \mathrm{~F}_{5}\right): 811,797,(\mathrm{Au}-$ S) 358. ${ }^{1} \mathrm{H} \mathrm{NMR}\left(\mathrm{CD}_{3}\right)_{2} \mathrm{CO}: \delta 6.07\left(\mathrm{br} \mathrm{s}, 1 \mathrm{H}, \mathrm{NH}_{2}\right), 3.48(\mathrm{~m}$, $\left.2 \mathrm{H}, \mathrm{CH}_{2}-\mathrm{N}\right), 2.82\left(\mathrm{~m}, 2 \mathrm{H}, \mathrm{CH}_{2}-\mathrm{S}\right)$ ppm. ${ }^{19} \mathrm{~F}$ NMR $\left(\mathrm{CD}_{3}\right)_{2} \mathrm{CO}: \delta-122.4(\mathrm{~m}, 2 \mathrm{~F}, o-\mathrm{F}),-124.3(\mathrm{~m}, 2 \mathrm{~F}, o-\mathrm{F})$, $-160.8\left(\mathrm{t}, 1 \mathrm{~F}, J_{p-\mathrm{F}, m-\mathrm{F}}=19.4 \mathrm{~Hz}, p-\mathrm{F}\right),-161.1\left(\mathrm{t}, 1 \mathrm{~F}, J_{p-\mathrm{F}, m-\mathrm{F}}=\right.$ $19.4 \mathrm{~Hz}, p-\mathrm{F}),-164.8(\mathrm{~m}, 2 \mathrm{~F}, m-\mathrm{F}),-165.6(\mathrm{~m}, 2 \mathrm{~F}, m-\mathrm{F})$ ppm. ${ }^{13} \mathrm{C}\left\{{ }^{1} \mathrm{H}\right\} \operatorname{NMR}\left(\mathrm{CD}_{3}\right)_{2} \mathrm{CO}: \delta 56.4\left(\mathrm{~s}, 1 \mathrm{C}, \mathrm{CH}_{2}-\mathrm{N}\right), 31.6$ (s, 1C, $\left.\mathrm{CH}_{2}-\mathrm{S}\right)$ ppm. MS $\left(\mathrm{ESI}^{+}\right) \mathrm{m} / z(\%): 608$ (76) $[\mathrm{M}+$ $\mathrm{H}]^{+}$.

Synthesis of Complex 3. A solution of $\left[\mathrm{Au}(\mathrm{OTf})\left(\mathrm{PPh}_{3}\right)\right]$ $(0.0605 \mathrm{~g}, 0.1 \mathrm{mmol})$ prepared in situ and [Au$\left.\left(\mathrm{C}_{6} \mathrm{~F}_{5}\right)_{2}\left(\mathrm{SC}_{6} \mathrm{H}_{4} \mathrm{NH}_{2}\right)\right]$ (1) $(0.0655 \mathrm{~g}, 0.1 \mathrm{mmol})$ was stirred in dichloromethane $(20 \mathrm{~mL})$ for $2 \mathrm{~h}$. The volume was reduced to $5 \mathrm{~mL}$, and addition of hexane afforded compound 3 as a yellow solid which was finally filtered. Yield: $0.088 \mathrm{~g}, 70 \%$. Anal. Calcd for $\mathrm{C}_{37} \mathrm{H}_{21} \mathrm{Au}_{2} \mathrm{~F}_{13} \mathrm{NO}_{3} \mathrm{PS}_{2}$ (1263.58): C, 35.17; $\mathrm{H}$, $1.68 ; \mathrm{N}, 1.11 ; \mathrm{S}, 5.08$. Found: C, 34.80; H, 2.09; N, 1.43; S, 4.85. IR $\left(\mathrm{cm}^{-1}\right):\left(\mathrm{NH}_{2}\right): 3056 ;\left(\mathrm{C}_{6} \mathrm{~F}_{5}\right): 1507,964 ;\left(\right.$ cis- $\left.\mathrm{C}_{6} \mathrm{~F}_{5}\right)$ : 812,$798 ; \nu_{\text {asym }}\left(\mathrm{SO}_{3}\right)=1272 ; \nu_{\text {sym }}\left(\mathrm{CF}_{3}\right)=1240 ; \nu_{\text {asym }}\left(\mathrm{CF}_{3}\right)=$ $1170 ; \nu_{\text {sym }}\left(\mathrm{SO}_{3}\right)=1025 ;(\mathrm{Au}-\mathrm{S}) 402,328 .{ }^{1} \mathrm{H} \quad \mathrm{NMR}$ $\left(\mathrm{CD}_{3}\right)_{2} \mathrm{CO}: \delta 8.25$ (br s, $\left.2 \mathrm{H}, \mathrm{NH}_{2}\right), 7.61\left(\mathrm{~m}, 15 \mathrm{H}, \mathrm{PPh}_{3}\right)$, $7.49(\mathrm{~m}, 1 \mathrm{H}, 6-\mathrm{H}), 7.36\left(\mathrm{~d}, 1 \mathrm{H}, 3 J_{\mathrm{H}-\mathrm{H}}=7.9 \mathrm{~Hz}, 3-\mathrm{H}\right), 7.24$ (m, 1H, 5-H), 7.09 (m, 1H, 4-H) ppm. ${ }^{31} \mathrm{P}\left\{{ }^{1} \mathrm{H}\right\}$ NMR $\left(\mathrm{CD}_{3}\right)_{2} \mathrm{CO}: \delta 34.8\left(\mathrm{~m}, 1 \mathrm{P}, \mathrm{PPh}_{3}\right) .{ }^{19} \mathrm{~F} \mathrm{NMR}\left(\mathrm{CD}_{3}\right)_{2} \mathrm{CO}: \delta$ $-78.7\left(\mathrm{~s}, 3 \mathrm{~F}, \mathrm{CF}_{3}\right),-121.2(\mathrm{~m}, 2 \mathrm{~F}, o-\mathrm{F}),-122.3(\mathrm{~m}, 2 \mathrm{~F}, o-\mathrm{F})$, $-158.1\left(\mathrm{t}, 1 \mathrm{~F}, J_{p-\mathrm{F}, m-\mathrm{F}}=19.1 \mathrm{~Hz}, p-\mathrm{F}\right),-158.3\left(\mathrm{t}, 1 \mathrm{~F}, J_{p-\mathrm{F}, m-\mathrm{F}}=\right.$ $19.4 \mathrm{~Hz}, p-\mathrm{F}),-162.9(\mathrm{~m}, 2 \mathrm{~F}, m-\mathrm{F}),-163.2(\mathrm{~m}, 2 \mathrm{~F}, m-\mathrm{F})$ ppm. ${ }^{13} \mathrm{C}\left\{{ }^{1} \mathrm{H}\right\}$ NMR $\left(\mathrm{CD}_{3}\right)_{2} \mathrm{CO}: \delta 135.0\left(\mathrm{~d}, 4 \mathrm{C}, J_{\mathrm{C}-\mathrm{P}}=13.8\right.$ $\left.\mathrm{Hz}, o-\mathrm{C}, \mathrm{PPh}_{3}\right), 133.4\left(\mathrm{~d}, 2 \mathrm{C}, J_{\mathrm{C}-\mathrm{P}}=2.4 \mathrm{~Hz}, p-\mathrm{C}, \mathrm{PPh}_{3}\right), 130.6$ $\left(\mathrm{d}, 4 \mathrm{C}, J_{\mathrm{C}-\mathrm{P}}=12.0 \mathrm{~Hz}, m-\mathrm{C}, \mathrm{PPh}_{3}\right), 130.6(\mathrm{~s}, 1 \mathrm{C}, 6-\mathrm{C}), 129.6$ (s, 1C, 5-C), 128.0 (s, 1C, 3-C), 125.4 (s, 1C, 4-C) ppm. MS $\left(\mathrm{ESI}^{+}\right) \mathrm{m} / z(\%): 1114(11)[\mathrm{M}-\mathrm{OTf}]^{+}$.

Synthesis of Complex 4. To an acetone solution $(20 \mathrm{~mL})$ of $\left[\mathrm{Au}\left(\mathrm{C}_{6} \mathrm{~F}_{5}\right)_{2}\left(\mathrm{SC}_{6} \mathrm{H}_{4} \mathrm{NH}_{2}\right)\right](1)(0.0656 \mathrm{~g}, 0.1 \mathrm{mmol})$ was added $\mathrm{Ag}(\mathrm{OTf})(0.0257 \mathrm{~g}, 0.1 \mathrm{mmol})$. After the addition, the reaction mixture was stirred for $1 \mathrm{~h}$. The volume was reduced to $5 \mathrm{~mL}$, and addition of hexane afforded compound 4 as a white solid which was finally filtered. Yield: $0.062 \mathrm{~g}$, 68\%. Anal. Calcd for $\mathrm{C}_{19} \mathrm{H}_{6} \mathrm{AuAgF}_{13} \mathrm{NO}_{3} \mathrm{~S}_{2}$ (912.20): C, 25.02; H, 0.66; N, 1.54; S, 7.03. Found: C, 25.07; H, 0.54; N, 1.51; S, 7.02. IR $\left(\mathrm{cm}^{-1}\right):\left(\mathrm{NH}_{2}\right): 3218 ;\left(\mathrm{C}_{6} \mathrm{~F}_{5}\right): 1508,965 ;\left(\right.$ cis- $\left.\mathrm{C}_{6} \mathrm{~F}_{5}\right): 814$, $800 ; \nu_{\text {asym }}\left(\mathrm{SO}_{3}\right)=1265 ; \nu_{\text {sym }}\left(\mathrm{CF}_{3}\right)=1223 ; \nu_{\text {asym }}\left(\mathrm{CF}_{3}\right)=$ $1169 ; \nu_{\text {sym }}\left(\mathrm{SO}_{3}\right)=1022 ;(\mathrm{Au}-\mathrm{S}) 350 .{ }^{1} \mathrm{H} \mathrm{NMR}\left(\mathrm{CD}_{3}\right)_{2} \mathrm{CO}: \delta$ 8.21 (br s, $\left.2 \mathrm{H}, \mathrm{NH}_{2}\right), 7.50(\mathrm{~m}, 1 \mathrm{H}, 6-\mathrm{H}), 7.38(\mathrm{~m}, 1 \mathrm{H}, 3-\mathrm{H})$, $7.24(\mathrm{~m}, 1 \mathrm{H}, 5-\mathrm{H}), 7.11(\mathrm{~m}, 1 \mathrm{H}, 4-\mathrm{H})$ ppm. ${ }^{19} \mathrm{~F}$ NMR $\left(\mathrm{CD}_{3}\right)_{2} \mathrm{CO}: \delta-80.0\left(\mathrm{~s}, 3 \mathrm{~F}, \mathrm{CF}_{3}\right),-122.5(\mathrm{~m}, 2 \mathrm{~F}, o-\mathrm{F}),-123.7$ $(\mathrm{m}, 2 \mathrm{~F}, o-\mathrm{F}),-159.5(\mathrm{~m}, 1 \mathrm{~F}, p-\mathrm{F}),-159.8(\mathrm{~m}, 1 \mathrm{~F}, p-\mathrm{F})$, $-164.4(\mathrm{~m}, 2 \mathrm{~F}, m-\mathrm{F}),-164.7(\mathrm{~m}, 2 \mathrm{~F}, m-\mathrm{F}) \mathrm{ppm} .{ }^{13} \mathrm{C}\left\{{ }^{1} \mathrm{H}\right\}$ NMR ( $\left.\mathrm{CD}_{3}\right)_{2} \mathrm{CO}: \delta 131.7$ (s, 1C, 6-C), 130.0 (s, 1C, 5-C), 128.3 (s, 1C, 3-C), 127.0 (s, 1C, 4-C) ppm. MS $\left(\mathrm{ESI}^{+}\right) \mathrm{m} / \mathrm{z}$ (\%): $762(79)[\mathrm{M}-\mathrm{OTf}]^{+}$.

Synthesis of Complex 5. To a dichloromethane solution $(20 \mathrm{~mL})$ of $\left[\mathrm{Au}\left(\mathrm{C}_{6} \mathrm{~F}_{5}\right)_{2}\left(\mathrm{SCH}_{2} \mathrm{CH}_{2} \mathrm{NH}_{2}\right)\right]$ (2) (0.0607 g, 0.1 $\mathrm{mmol})$ was added $\left[\mathrm{Au}\left(\mathrm{C}_{6} \mathrm{~F}_{5}\right)_{3}(\mathrm{tht})\right](0.079 \mathrm{~g}, 0.1 \mathrm{mmol})$. After the addition, the reaction mixture was stirred for $1 \mathrm{~h}$. The volume was reduced to $5 \mathrm{~mL}$, and addition of hexane afforded compound $\mathbf{5}$ as a yellow solid which was finally filtered. Yield: 0.044 g, 45\%. Anal. Calcd for $\mathrm{C}_{20} \mathrm{H}_{6} \mathrm{Au}_{2} \mathrm{~F}_{15} \mathrm{NS}$ (971.24): C, 24.73; H, 0.62; N, 1.44; S, 3.30. Found: C, 24.51; H, 0.97; N, 1.58 ; S, 3.58. IR $\left(\mathrm{cm}^{-1}\right):\left(\mathrm{NH}_{2}\right): 3334,3283 ;\left(\mathrm{Au}^{\mathrm{III}}-\mathrm{C}_{6} \mathrm{~F}_{5}\right)$ : 1505, 959; $\left(\right.$ cis $\left.-\mathrm{C}_{6} \mathrm{~F}_{5}\right): 815,794 ;\left(\mathrm{Au}^{\mathrm{I}}-\mathrm{C}_{6} \mathrm{~F}_{5}\right): 1485,948,794$; $(\mathrm{Au}-\mathrm{S}) 358,351 .{ }^{1} \mathrm{H}$ NMR $\left(\mathrm{CD}_{3}\right)_{2} \mathrm{CO}: \delta 6.36($ br s, $2 \mathrm{H}$, 
$\mathrm{NH}_{2}$ ), $3.83\left(\mathrm{~m}, 2 \mathrm{H}, \mathrm{CH}_{2}-\mathrm{N}\right), 3.52\left(\mathrm{~m}, 2 \mathrm{H}, \mathrm{CH}_{2}-\mathrm{S}\right) \mathrm{ppm} .{ }^{19} \mathrm{~F}$ NMR $\left(\mathrm{CD}_{3}\right)_{2} \mathrm{CO}: \delta-116.8\left(\mathrm{~m}, 2 \mathrm{~F}, o-\mathrm{F}, \mathrm{Au}^{\mathrm{I}}\right),-161.4(\mathrm{t}, 1 \mathrm{~F}$, $\left.J_{p-\mathrm{F}, m-\mathrm{F}}=19.6 \mathrm{~Hz}, p-\mathrm{F}, \mathrm{Au}^{\mathrm{I}}\right),-162.9\left(\mathrm{~m}, 2 \mathrm{~F}, m-\mathrm{F}, \mathrm{Au}^{\mathrm{I}}\right),-120.3$ $\left(\mathrm{m}, 2 \mathrm{~F}, J_{o-\mathrm{F}, m-\mathrm{F}}=21.4 \mathrm{~Hz}, o-\mathrm{F}, \mathrm{Au}^{\mathrm{III}}\right),-122.8(\mathrm{~m}, 2 \mathrm{~F}, o-\mathrm{F}$, $\left.\mathrm{Au}^{\mathrm{III}}\right),-157.7\left(\mathrm{t}, 1 \mathrm{~F}, J_{p-\mathrm{F}, m-\mathrm{F}}=19.3 \mathrm{~Hz}, p-\mathrm{F}, \mathrm{Au}^{\mathrm{III}}\right),-158.0(\mathrm{t}$, $\left.1 \mathrm{~F}, J_{p-\mathrm{F}, m-\mathrm{F}}=19.4 \mathrm{~Hz}, p-\mathrm{F}, \mathrm{Au}^{\mathrm{III}}\right),-163.1\left(\mathrm{~m}, 2 \mathrm{~F}, m-\mathrm{F}, \mathrm{Au}^{\mathrm{III}}\right)$, $-164.3\left(\mathrm{~m}, 2 \mathrm{~F}, m-\mathrm{F}, \mathrm{Au}{ }^{\mathrm{III}}\right) \mathrm{ppm} .{ }^{13} \mathrm{C}\left\{{ }^{1} \mathrm{H}\right\} \mathrm{NMR}\left(\mathrm{CD}_{3}\right)_{2} \mathrm{CO}: \delta$ 55.9 (s, 1C, $\mathrm{CH}_{2}-\mathrm{N}$ ), 35,8 (s, 1C, $\left.\mathrm{CH}_{2}-\mathrm{S}\right)$ ppm. MS $\left(\mathrm{ESI}^{+}\right)$ $m / z(\%): 994$ (4) $[\mathrm{M}+\mathrm{Na}]^{+}$.

General Procedure of the Synthesis of the Complexes 6-8. A mixture of $\left[\mathrm{Au}\left(\mathrm{C}_{6} \mathrm{~F}_{5}\right)_{2}\left(\mathrm{SC}_{6} \mathrm{H}_{4} \mathrm{NH}_{2}\right)\right]$ (1) (0.1966 g, $0.3 \mathrm{mmol}$ ) with CN-cyclohexyl (0.0373 g, 0.3 mmol) (6) or CN-naphtyl (0.0460 g, $0.3 \mathrm{mmol})(7)$, or $\left[\mathrm{Au}\left(\mathrm{C}_{6} \mathrm{~F}_{5}\right)_{2}\left(\mathrm{SCH}_{2} \mathrm{CH}_{2} \mathrm{NH}_{2}\right)\right](2)(0.1882 \mathrm{~g}, 0.3 \mathrm{mmol})$ with $\mathrm{CN}$-cyclohexyl $(0.0373 \mathrm{~g}, 0.3 \mathrm{mmol})(8)$ in dichloromethane $(20 \mathrm{~mL})$, was stirred for $24 \mathrm{~h}(6,7)$ or $72 \mathrm{~h}(8)$. The volume was reduced to $5 \mathrm{~mL}$, and addition of $n$-hexane afforded 6-8 as off-white solids which were finally filtered.

Complex 6. Yield: $0.134 \mathrm{~g}, 58 \%$. Anal. Calcd for $\mathrm{C}_{25} \mathrm{H}_{17} \mathrm{AuF}_{10} \mathrm{~N}_{2} \mathrm{~S}$ (764.43): C, 39.28; H, 2.24; N, 3.66; S, 4.19. Found: C, 38.97; H, 2.26; N, 3.79; S, 4.32. IR $\left(\mathrm{cm}^{-1}\right)$ : $\left(\mathrm{NH}_{2}\right): 3400,3363 ; \nu(\mathrm{C}=\mathrm{N}): 1558 ;\left(\mathrm{C}_{6} \mathrm{~F}_{5}\right): 1506,955 ;$ (cis$\left.\mathrm{C}_{6} \mathrm{~F}_{5}\right):$ 800, 791; $(\mathrm{Au}-\mathrm{S})$ 403. ${ }^{1} \mathrm{H}$ NMR $\left(\mathrm{CD}_{3}\right)_{2} \mathrm{CO}: \delta 9.94$ (br s, 1H, NH), $7.98\left(\mathrm{~m}, 1 \mathrm{H}, \mathrm{NH}_{\mathrm{Cy}}\right), 7.47(\mathrm{~m}, 1 \mathrm{H}, 6-\mathrm{H}), 7.31$ (m, 1H, 3-H), $7.14(\mathrm{~m}, 1 \mathrm{H}, 4-\mathrm{H}), 7.06(\mathrm{~m}, 1 \mathrm{H}, 5-\mathrm{H}), 4.20(\mathrm{~m}$, $\left.1 \mathrm{H}, \mathrm{CH}_{\mathrm{Cy}}\right), 1.99-1.14\left(\mathrm{~m}, 10 \mathrm{H}, \mathrm{CH}_{2 \mathrm{Cy}}\right)$ ppm. ${ }^{19} \mathrm{~F} \mathrm{NMR}$ $\left(\mathrm{CD}_{3}\right)_{2} \mathrm{CO}: \delta-121.4(\mathrm{~m}, 2 \mathrm{~F}, o-\mathrm{F}),-123.5(\mathrm{~m}, 2 \mathrm{~F}, o-\mathrm{F})$, $-160.8\left(\mathrm{t}, 1 \mathrm{~F}, J_{p-\mathrm{F}, m-\mathrm{F}}=19.6 \mathrm{~Hz}, p-\mathrm{F}\right),-162.2\left(\mathrm{t}, 1 \mathrm{~F}, J_{p-\mathrm{F}, m-\mathrm{F}}=\right.$ $19.3 \mathrm{~Hz}, p-\mathrm{F}),-164.8(\mathrm{~m}, 2 \mathrm{~F}, m-\mathrm{F}),-165.6(\mathrm{~m}, 2 \mathrm{~F}, m-\mathrm{F})$ ppm. ${ }^{13} \mathrm{C}\left\{{ }^{1} \mathrm{H}\right\}$ NMR $\left(\mathrm{CD}_{3}\right)_{2} \mathrm{CO}: \delta 132.4$ (s, 1C, 6-C), 126.5 (s, 1C, 5-C), 126.3 (s, 1C, 4-C), 122.6 (s, 1C, 3-C), 54.3 (s, $\left.1 \mathrm{C}, \mathrm{CH}_{\mathrm{Cy}}\right), 32.3\left(\mathrm{~s}, \mathrm{CH}_{2 \mathrm{C}_{y}}\right), 25.6\left(\mathrm{~s}, \mathrm{CH}_{2 \mathrm{Cy}}\right), 25.3\left(\mathrm{~s}, \mathrm{CH}_{2 \mathrm{Cy}}\right)$ ppm. MS $\left(\mathrm{ESI}^{+}\right) \mathrm{m} / z(\%): 787(51)[\mathrm{M}+\mathrm{Na}]^{+}$.

Complex 7. Yield: $0.053 \mathrm{~g}, 22 \%$. Anal. Calcd for $\mathrm{C}_{29} \mathrm{H}_{13} \mathrm{AuF}_{10} \mathrm{~N}_{2} \mathrm{~S}$ (808.45): C, 43.08; H, 1.62; N, 3.47; S, 3.97. Found: C, 43.10; H, 1.70; N, 3.52; S, 4.03. IR $\left(\mathrm{cm}^{-1}\right)$ : $\left(\mathrm{NH}_{2}\right): 3049 ; \nu(\mathrm{C}=\mathrm{N}): 1571 ;\left(\mathrm{C}_{6} \mathrm{~F}_{5}\right): 1506,964 ;\left(\right.$ cis- $\left.\mathrm{C}_{6} \mathrm{~F}_{5}\right)$ : 805, 787; (Au-S) 371. ${ }^{1} \mathrm{H}$ NMR $\left(\mathrm{CD}_{3}\right)_{2} \mathrm{CO}: \delta 9.72($ br s, $1 \mathrm{H}$, $\mathrm{NH}), 8.66(\mathrm{~m}, 1 \mathrm{H}$, naphthyl), 7.89 (m, 1H, naphthyl), 7.86 (m, 2H, naphthyl), $7.78(\mathrm{~m}, 1 \mathrm{H}, 6-\mathrm{H}), 7.75(\mathrm{~m}, 1 \mathrm{H}$, naphthyl), $7.70(\mathrm{~m}, 1 \mathrm{H}, 3-\mathrm{H}), 7.49$ (m, 1H, naphthyl), 7.38 (m, 2H, 4-H, naphthyl), $7.20(\mathrm{~m}, 1 \mathrm{H}, 5-\mathrm{H}) \mathrm{ppm} .{ }^{19} \mathrm{~F}$ NMR $\left(\mathrm{CD}_{3}\right)_{2} \mathrm{CO}: \delta$ $-117.0(\mathrm{~m}, 4 \mathrm{~F}, o-\mathrm{F}),-165.9(\mathrm{~m}, 2 \mathrm{~F}, p-\mathrm{F}),-166.8(\mathrm{~m}, 4 \mathrm{~F}, m-$ F) ppm. ${ }^{13} \mathrm{C}\left\{{ }^{1} \mathrm{H}\right\} \operatorname{NMR}\left(\mathrm{CD}_{3}\right)_{2} \mathrm{CO}: \delta 155.4(\mathrm{~s}, 1 \mathrm{C}), 139.4(\mathrm{~s}$, 1C), 135.3 (s, 1C), 129.6 (s, 1C, naphthyl), 128.5 (s, 1C, naphthyl), 128.1 (s, 1C, naphthyl), 127.4 (s, 1C, naphthyl), 126.8 (s, 1C, naphthyl), 125.1 (s, 1C, 4-C), 123.5 (s, 1C, 5-C), 121.7 (s, 1C, 6-C or naphthyl), 120.7 (s, 1C, 3-C), 120.3 (s, 1C 6-C or naphthyl), 114.6 (s, 1C, naphthyl) ppm.

Complex 8. Yield: $0.089 \mathrm{~g}, 41 \%$. Anal. Calcd for $\mathrm{C}_{21} \mathrm{H}_{17} \mathrm{AuF}_{10} \mathrm{~N}_{2} \mathrm{~S}$ (716.39): C, 35.21; H, 2.39; N, 3.91; S, 4.48. Found: C, 34.98; H, 2.60; N, 3.91; S, 4.74. IR $\left(\mathrm{cm}^{-1}\right)$ : $\left(\mathrm{NH}_{2}\right): 3426,(\mathrm{C}=\mathrm{N}): 1580,\left(\mathrm{C}_{6} \mathrm{~F}_{5}\right): 1505$, 959; $\left(\right.$ cis- $\left.\mathrm{C}_{6} \mathrm{~F}_{5}\right)$ : 800, 792; ( $\mathrm{Au}-\mathrm{S}) 366 .{ }^{1} \mathrm{H} \mathrm{NMR}\left(\mathrm{CD}_{3}\right)_{2} \mathrm{CO}: \delta 8.57(\mathrm{br} \mathrm{s}, 1 \mathrm{H}$, $\mathrm{NH}), 7.49\left(\right.$ br s, $\left.1 \mathrm{H}, \mathrm{NH}_{\mathrm{Cy}}\right), 3.69\left(\mathrm{~m}, 2 \mathrm{H}, \mathrm{CH}_{2}-\mathrm{N}\right), 3.48(\mathrm{~m}$, $\left.1 \mathrm{H}, \mathrm{CH}_{\mathrm{Cy}}\right), 2.67\left(\mathrm{~m}, 2 \mathrm{H}, \mathrm{CH}_{2}-\mathrm{S}\right), 1.85-1.24(\mathrm{~m}, 10 \mathrm{H}$, $\mathrm{CH}_{2 \mathrm{Cy}}$ ) ppm. ${ }^{19} \mathrm{~F}$ NMR $\left(\mathrm{CD}_{3}\right)_{2} \mathrm{CO}: \delta-122.1$ (m, 2F, o-F), $-123.0(\mathrm{~m}, 2 \mathrm{~F}, o-\mathrm{F}),-161.6\left(\mathrm{t}, 1 \mathrm{~F}, J_{p-\mathrm{F}, m-\mathrm{F}}=19.5 \mathrm{~Hz}, p-\mathrm{F}\right)$, $-163.0\left(\mathrm{t}, 1 \mathrm{~F}, J_{p-\mathrm{F}, m-\mathrm{F}}=19.3 \mathrm{~Hz}, p-\mathrm{F}\right),-165.0(\mathrm{~m}, 2 \mathrm{~F}, m-\mathrm{F})$, $-166.0(\mathrm{~m}, 2 \mathrm{~F}, \mathrm{~m}-\mathrm{F}) \mathrm{ppm} .{ }^{13} \mathrm{C}\left\{{ }^{1} \mathrm{H}\right\} \mathrm{NMR}\left(\mathrm{CD}_{3}\right)_{2} \mathrm{CO}: \delta 55.3$ (s, 1C, $\mathrm{CH}_{2}-\mathrm{N}$ ), 53.4 (s, 1C, $\mathrm{CH}_{\mathrm{Cy}}$ ), $32.2\left(\mathrm{~s}, 1 \mathrm{C}, \mathrm{CH}_{2 \mathrm{Cy}}\right), 27.4$ (s, 1C, $\mathrm{CH}_{2}-\mathrm{S}$ ), 25.8 (s, 1C, $\mathrm{CH}_{2 \mathrm{Cy}}$ ), 32.3 (s, 1C, $\mathrm{CH}_{2 C_{y}}$ ), 25.6 (s, 1C, $\left.\mathrm{CH}_{2 C_{y}}\right), 25.3\left(\mathrm{~s}, 1 \mathrm{C}, \mathrm{CH}_{2 \mathrm{Cy}}\right)$ ppm. MS $\left(\mathrm{ESI}^{+}\right) \mathrm{m} / z$ (\%): $717(100)[\mathrm{M}+\mathrm{H}]^{+}$.

Crystallography. Data were registered on a Bruker SMART 1000 CCD diffractometer. The crystals were mounted on glass fibers using inert oil and transferred to the cold gas stream of the diffractometer. Data were collected using monochromated $\operatorname{MoK} \alpha$ radiation $(\lambda=0.71073)$ in $\omega$ scans. Absorption corrections based on multiple scans were applied with the program SADABS. The structures were solved by direct methods and refined on $F^{2}$ using the program SHELXL2016. ${ }^{18}$ All nonhydrogen atoms were refined anisotropically. Hydrogen atoms were included using a riding model. Two different crystals in a 95.5:4.5 ratio were identified in compound 5. Only the four gold atoms of the minority crystal could be detected. Structure was refined as two fragments with the Part instruction. CCDC deposition numbers 1846294 (5) and 1846295 (8) contain the supplementary crystallographic data. These data can be obtained free of charge from The Cambridge Crystallography Data Center.

\section{ASSOCIATED CONTENT}

\section{Supporting Information}

The Supporting Information is available free of charge on the ACS Publications website at DOI: 10.1021/acsomega.8b01547.

Crystallographic data of complex 5 (CIF)

Crystallographic data of complex 8 (CIF)

\section{AUTHOR INFORMATION}

\section{Corresponding Authors}

*E-mail: dvilla@unizar.es (M.D.V.).

*E-mail: gimeno@unizar.es (M.C.G.).

ORCID $\odot$

M. Concepción Gimeno: 0000-0003-0553-0695

Notes

The authors declare no competing financial interest.

\section{ACKNOWLEDGMENTS}

This work is dedicated to Professor Ernesto Carmona in recognition of his outstanding contribution to modern organometallic chemistry. Authors thank the Ministerio de Economía y Competitividad (MINECO/FEDER CTQ201675816-C2-1-P and Gobierno de Aragón-Fondo Social Europeo (E07-17R) for the financial support of our research.

\section{REFERENCES}

(1) (a) Gimeno, M. C.; Laguna, A. Comprehensive Coordination Chemistry II; Silver and Gold; McCleverty, J. A., Meyer, T. J., Eds.; Elsevior: Oxford, 2004; Vol. 6, pp 911-1145; (b) Gimeno, M. C. The Chemistry of Gold in Modern Supramolecular Gold Chemistry. GoldMetal Interactions and Applications, Laguna, A.; Ed.; Wiley-VCH, 2008, pp 1-63.

(2) (a) Hashmi, A. S. K.; Hutchings, G. J. Gold Catalysis. Angew. Chem., Int. Ed. 2006, 45, 7896-7936. (b) Hashmi, A. S. K. GoldCatalyzed Organic Reactions. Chem. Rev. 2007, 107, 3180-3211. (c) Hanhan, M. E. Novel cycloaurated gold(III) complexes and their effects on styrene polymerization. Gold Bull. 2011, 44, 43-47. (d) Mankad, N. P.; Toste, F. D. C-C Coupling Reactivity of an Alkylgold(III) Fluoride Complex with Arylboronic Acids. J. Am. Chem. Soc. 2010, 132, 12859-12861. (e) Pflästerer, D.; Hashmi, A. S. K. Gold catalysis in total synthesis-recent achievements. Chem. Soc. Rev. 2016, 45, 1331-1367. 
(3) (a) Yam, V. W.-W.; Cheng, E. C.-C. Photochemistry and Photophysics of Coordination Compounds: Gold. Top. Curr. Chem. 2007, 281, 269-309. (b) Visbal, R.; Gimeno, M. C. N-heterocyclic carbene metal complexes: photoluminescence and applications. Chem. Soc. Rev. 2014, 43, 3551-3574.

(4) (a) Tiekink, E. R. T. Gold derivatives for the treatment of cancer. Crit. Rev. Oncol. Hematol. 2002, 42, 225-248. (b) Henderson, W. The Chemistry of Cyclometallated Gold(III) Complexes with C,N-Donor Ligands. Adv. Organomet. Chem. 2006, 54, 207-265. (c) Bertrand, B.; Casini, A. A golden future in medicinal inorganic chemistry: the promise of anticancer gold organometallic compounds. Dalton Trans. 2014, 43, 4209-4219. (d) Zou, T.; Lum, C. T.; Lok, C.-N.; Zhang, J.J.; Che, C.-M. Chemical biology of anticancer gold(III) and gold(I) complexes. Chem. Soc. Rev. 2015, 44, 8786-8801. (e) Sun, R. W.-Y.; Ma, D.-L.; Wong, E. L.-M.; Che, C.-M. Some uses of transition metal complexes as anti-cancer and anti-HIV agents. Dalton Trans. 2007, 4884-4892.

(5) (a) Casado, R.; Contel, M.; Laguna, M.; Romero, P.; Sanz, S. Organometallic Gold(III) Compounds as Catalysts for the Addition of Water and Methanol to Terminal Alkynes. J. Am. Chem. Soc. 2003, 125, 11925-11935. (b) González-Arellano, C.; Corma, A.; Iglesias, M.; Sánchez, F. Homogeneous and heterogenized Au(III) Schiff basecomplexes as selective and general catalysts for self-coupling of aryl boronic acids. Chem. Commun. 2005, 1990-1992. (c) Lo, V. K.-Y.; Liu, Y.; Wong, M.-K.; Che, C.-M. Gold(III) Salen Complex-Catalyzed Synthesis of Propargylamines via a Three-Component Coupling Reaction. Org. Lett. 2006, 8, 1529-1532. (d) Oña-Burgos, P.; Fernández, I.; Roces, L.; Fernández, L. T.; García-Granda, S.; Ortiz, F. L. An Unprecedented Phosphinamidic Gold(III) Metallocycle: Synthesis via Tin(IV) Precursors, Structure, and Multicomponent Catalysis. Organometallics 2009, 28, 1739-1747. (e) Pažický, M.; Loos, A.; Ferreira, M. J.; Serra, D.; Vinokurov, N.; Rominger, F.; Jäkel, C.; Hashmi, A. S. K.; Limbach, M. Synthesis, Reactivity, and Electrochemical Studies of Gold(I) and Gold(III) Complexes Supported by N-Heterocyclic Carbenes and Their Application in Catalysis. Organometallics 2010, 29, 4448-4458.

(6) (a) Bronner, C.; Wenger, O. S. Luminescent cyclometalated gold(III) complexes. Dalton Trans. 2011, 40, 12409-12420. (b) Au, V. K.-M.; Wong, K. M.-C.; Zhu, N.; Yam, V. W.-W. Luminescent Cyclometalated Dialkynylgold(III) Complexes of 2-PhenylpyridineType Derivatives with Readily Tunable Emission Properties. Chem.Eur. J. 2011, 17, 130-142. (c) Tang, M.-C.; Tsang, D. P.-K.; Wong, Y.-C.; Chan, M.-Y.; Wong, K. M.-C.; Yam, V. W.-W. Bipolar Gold(III) Complexes for Solution-Processable Organic LightEmitting Devices with a Small Efficiency Roll-Off. J. Am. Chem. Soc. 2014, 136, 17861-17868. (d) Gimeno, M. C.; López-de-Luzuriaga, J. M.; Manso, E.; Monge, M.; Olmos, M. E.; Rodríguez-Castillo, M.; Tena, M.-T.; Day, D. P.; Lawrence, E. J.; Wildgoose, G. G. Synthesis, Photochemical, and Redox Properties of Gold(I) and Gold(III) Pincer Complexes Incorporating a 2,2':6',2'-Terpyridine Ligand Framework. Inorg. Chem. 2015, 54, 10667-10677. (e) Tsai, J. L.-L.; Chan, A. O.-Y.; Che, C.-M. A luminescent cyclometalated gold(iii)avidin conjugate with a long-lived emissive excited state that binds to proteins and DNA and possesses anti-proliferation capacity. Chem. Commun. 2015, 51, 8547-8550. (f) Fernández-Cestau, J.; Giménez, N.; Lalinde, E.; Montaño, P.; Moreno, M. T.; Sánchez, S.; Weber, M. D.; Costa, R. D. Alkynyl bridged cyclometalated $\operatorname{Ir}_{2} \mathrm{M}_{2}$ clusters: impact of the heterometal in the photo- and electro-luminescence properties. Dalton Trans. 2016, 45, 3251-3255. (g) Currie, L.; Fernandez-Cestau, J.; Rocchigiani, L.; Bertrand, B.; Lancaster, S. J.; Hughes, D. L.; Duckworth, H.; Jones, S. T. E.; Credgington, D.; Penfold, T. J.; Bochmann, M. Luminescent Gold(III) Thiolates: Supramolecular Interactions Trigger and Control Switchable Photoemissions from Bimolecular Excited States. Chem.-Eur. J. 2017, 23, 105-113.

(7) (a) Marcon, G.; Carotti, S.; Coronnello, M.; Messori, L.; Mini, E.; Orioli, P.; Mazzei, T.; Cinellu, M. A.; Minghetti, G. Gold(III) Complexes with Bipyridyl Ligands: Solution Chemistry, Cytotoxicity, and DNA Binding Properties. J. Med. Chem. 2002, 45, 1672-1677. (b) Wang, X.; Guo, Z. Towards the rational design of platinum(II) and gold(III) complexes as antitumour agents. Dalton Trans. 2008, 1521-1532. (c) Yan, J. J.; Chow, A. L.-F.; Leung, C.-H.; Sun, R. W.Y.; Ma, D.-L.; Che, C.-M. Cyclometalated gold(III) complexes with $\mathrm{N}$-heterocyclic carbene ligands as topoisomerase I poisons. Chem.Commun. 2010, 46, 3893-3895. (d) Cutillas, N.; Yellol, G. S.; de Haro, C.; Vicente, C.; Rodríguez, V.; Ruiz, J. Anticancer cyclometalated complexes of platinum group metals and gold. Coord. Chem. Rev. 2013, 257, 2784-2797. (e) Sun, R. W.-Y.; Lok, C.-N.; Fong, T. T.-H.; Li, C. K.-L.; Yang, Z. F.; Zou, T.; Siu, A. F.-M.; Che, C.-M. A dinuclear cyclometalated gold(iii)-phosphine complex targeting thioredoxin reductase inhibits hepatocellular carcinoma in vivo. Chem. Sci. 2013, 4, 1979-1988. (f) Omae, I. Applications of fivemembered ring products of cyclometalation reactions as anticancer agents. Coord. Chem. Rev. 2014, 280, 84-95. (g) Bertrand, B.; Spreckelmeyer, S.; Bodio, E.; Cocco, F.; Picquet, M.; Richard, P.; Le Gendre, P.; Orvig, C.; Cinellu, M. A.; Casini, A. Exploring the potential of gold(III) cyclometallated compounds as cytotoxic agents: variations on the $\mathrm{C}^{\wedge} \mathrm{N}$ theme. Dalton Trans. 2015, 44, 11911-11918.

(8) (a) Cerrada, E.; Fernandez, E. J.; Jones, P. G.; Laguna, A.; Laguna, M.; Terroba, R. Synthesis and Reactivity of Trinuclear Gold(III) Dithiolate Complexes. X-ray Structure of $\left[\mathrm{Au}\left(\mathrm{C}_{6} \mathrm{~F}_{5}\right)\right.$ $\left.\left(\mathrm{S}_{2} \mathrm{C}_{6} \mathrm{H}_{4}\right)\right]_{3}$ and $\left[\mathrm{Au}\left(\mathrm{C}_{6} \mathrm{~F}_{5}\right)\left(\mathrm{S}_{2} \mathrm{C}_{6} \mathrm{H}_{4}\right)\left(\mathrm{SC}_{6} \mathrm{H}_{4} \mathrm{SPPh}_{3}\right)\right]$. Organometallics 1995, 14, 5537-5543. (b) Crespo, O.; Gimeno, M. C.; Jones, P. G.; Laguna, A.; Villacampa, M. D. Synthesis and characterisation of gold(I) and -(III) complexes with 1-methyl-2-sulfanyl-1,2-dicarba-closododecaborate. J. Chem. Soc. Dalton Trans. 1997, 2963-2968. (c) Vicente, J.; Chicote, M. T.; González-Herrero, P.; Jones, P. G.; Humphrey, M. G.; Cifuentes, M. P.; Samoc, M.; Luther-Davies, B. Complexes with S-Donor Ligands. 7. New 1,1-Ethylenedithiolato Complexes of Thallium(I), Gold(I), and Gold(III): Syntheses, Structure, and Molecular Cubic Hyperpolarizabilities $\dagger$. Inorg. Chem. 1999, 38, 5018-5026. (d) Nöth, H.; Beck, W.; Burger, K. The Molecular Structure of Some Transition-Metal Complexes with 1,2,3,4-Tetrazole-5-thiolate Anions. Eur. J. Inorg. Chem. 1998, 9399. (e) Ortner, K.; Abram, U. Gold(III) complexes with diphenylthiocarbazonate. Synthesis and structures of $[\mathrm{Au}(\mathrm{Hdamp}-$ $\left.\left.\mathrm{C}_{1}\right)\{\mathrm{PhNHNC}(\mathrm{S}) \mathrm{NNPh}\} \mathrm{Cl}\right] \mathrm{Cl} \times \mathrm{H}_{2} \mathrm{O}$ and $\left[\mathrm{Au}\left(\mathrm{Hdamp}-\mathrm{C}_{1}\right)-\right.$ $\{\mathrm{PhNNC}(\mathrm{S}) \mathrm{NNPh}\}$ (Smetetraz) $]$ (Hdamp- $\mathrm{C}_{1}=2$ - (dimethylammonium-methyl)phenyl;-Smetetraz $=2$-methylmercaptotetrazolate). Polyhedron 1999, 18, 749-754. (f) Bardají, M.; Calhorda, M. J.; Costa, P. J.; Jones, P. G.; Laguna, A.; Reyes Pérez, M.; Villacampa, M. D. Synthesis, Structural Characterization, and Theoretical Studies of Gold(I) and Gold(I)-Gold(III) Thiolate Complexes: Quenching of Gold(I) Thiolate Luminescence. Inorg. Chem. 2006, 45, 1059-1068.

(9) Bachman, R. E.; Bodolosky-Bettis, S. A.; Pyle, C. J.; Gray, M. A. Reversible Oxidative Addition and Reductive Elimination of Fluorinated Disulfides at Gold(I) Thiolate Complexes: A New Ligand Exchange Mechanism. J. Am. Chem. Soc. 2008, 130, 1430314310.

(10) (a) Canales, F.; Canales, S.; Crespo, O.; Gimeno, M. C.; Jones, P. G.; Laguna, A. Synthesis and Reactivity of the First (Hydrosulfido)gold(III) Complex. Crystal Structure of the Derivatives $\mathrm{NBu}_{4}[\{\mathrm{Au}-$ $\left.\left(\mathrm{C}_{6} \mathrm{~F}_{5}\right)_{3}\right\}_{2} \mathrm{SR}$ ] with the Isolobal Fragments $\mathrm{R}=\mathrm{H}, \mathrm{AuPPh}_{3}, \mathrm{AgPPh}_{3}$. Organometallics 1998, 17, 1617-1621. (b) Crespo, O.; Canales, F.; Gimeno, M. C.; Jones, P. G.; Laguna, A. Synthesis of $\left[\mathrm{Au}_{2}\left(\mathrm{SC}_{6} \mathrm{~F}_{5}\right)_{2}(\mu\right.$ $\mathrm{dppf})]$ and $\left[\mathrm{Au}_{2}\left(\mu-\mathrm{SC}_{6} \mathrm{~F}_{5}\right)(\mu\right.$-dppf $\left.)\right]\left(\mathrm{dppf}=1,1^{\prime}-\mathrm{Bis}-\right.$ (diphenylphosphino)ferrocene). Reactivity toward Various Metallic Fragments. Organometallics 1999, 18, 3142-3148.

(11) (a) Cinellu, M. A.; Minghetti, G.; Pinna, M. V.; Stoccoro, S.; Zucca, A.; Manassero, M. Replacement of the chloride ligand in $[\mathrm{Au}(\mathrm{C}, \mathrm{N}, \mathrm{N}) \mathrm{Cl}]\left[\mathrm{PF}_{6}\right]$ cyclometallated complexes by $\mathrm{C}, \mathrm{N}, \mathrm{O}$ and $\mathrm{S}$ donor anionic ligands. J. Chem. Soc., Dalton Trans. 1999, 2823-2831. (b) Henderson, W.; Nicholson, B. K.; Faville, S. J.; Fan, D.; Ranford, J. D. Gold(III) thiosalicylate complexes containing cycloaurated 2arylpyridine, 2-anilinopyridine and 2-benzylpyridine ligands. J. Organomet. Chem. 2001, 631, 41-46. (c) Fan, D.; Yang, C.-T.; Ranford, J. D.; Vittal, J. J.; Lee, P. F. Synthesis, characterization, and biological activities of 2-phenylpyridine gold(III) complexes with 
thiolate ligands. Dalton Trans. 2003, 3376-3381. (d) Casini, A.; Hartinger, C.; Gabbiani, C.; Mini, E.; Dyson, P. J.; Keppler, B. K.; Messori, L. Gold(III) compounds as anticancer agents: Relevance of gold-protein interactions for their mechanism of action. J. Inorg. Biochem. 2008, 102, 564-575. (e) Crespo, O.; Gimeno, M. C.; Jones, P. G.; Laguna, A.; Villacampa, M. D. Synthesis and characterisation of gold-(I) and -(III) complexes with 1-methyl-2-sulfanyl-1,2-dicarbacloso-dodecaborate. J. Chem. Soc., Dalton Trans. 1997, 2963-2968.

(12) (a) Slaughter, L. M. Acyclic Aminocarbenes in Catalysis. ACS Catal. 2012, 2, 1802-1816. (b) S. Handa, S.; Slaughter, L. M. Enantioselective Alkynylbenzaldehyde Cyclizations Catalyzed by Chiral Gold(I) Acyclic Diaminocarbene Complexes Containing Weak Au-Arene Interactions. Angew. Chem., Int. Ed. 2012, 51, 2912-2915. (c) Barbazanges, M.; Fensterbank, L. Chiral Acyclic Diaminocarbene Complexes: a New Opportunity for Gold Asymmetric Catalysis. ChemCatChem. 2012, 4, 1065-1066.

(13) (a) Minghetti, G.; Bonati, F. Bis(carbene) complexes of gold(I) and gold (III). J. Organomet. Chem. 1973, 54, C62-C63. (b) Minghetti, G.; Bonati, F.; Banditelli, G. Carbene complexes of gold(III) and reactions of the coordinated ligand. Inorg. Chem. 1976, 15, 1718-1720. (c) Usón, R.; Laguna, A.; Vicente, J.; García, J.; Bergareche, B.; Brun, P. Neutral isocyanide and carbene pentafluorophenyl complexes of gold(I) and gold(III). Inorg. Chim. Acta 1978, 28, 237-243. (d) Usón, R.; Laguna, A.; Villacampa, M. D. Mono and binuclear gold complexes with diamine carbene ligands. Inorg. Chim. Acta 1984, 81, 25-31. (e) Usón, R.; Laguna, A.; Villacampa, M. D.; Jones, P. G.; Sheldrick, G. M. Reactions of cis-diisocyanidebis(perfluorophenyl)gold(III) complexes with hydrazines. Crystal and molecular structure of cis- $\left[\mathrm{Au}\left\{\mathrm{C}\left(\mathrm{NHC}_{6} \mathrm{H}_{4} \mathrm{Me}-\mathrm{p}\right)=\mathrm{N}\right.\right.$ $\left.\left.\mathrm{N}(\mathrm{Ph}) \mathrm{C}\left(\mathrm{NHC}_{6} \mathrm{H}_{4} \mathrm{Me}-\mathrm{p}\right)\right\}\left(\mathrm{C}_{6} \mathrm{~F}_{5}\right)_{2}\right]$. J. Chem. Soc. Dalton Trans. 1984, 2035-2038. (f) Hashmi, A. S. K.; Hengst, T.; Lothschütz, C.; Rominger, F. New and Easily Accessible Nitrogen Acyclic Gold(I) Carbenes: Structure and Application in the Gold-Catalyzed Phenol Synthesis as well as the Hydration of Alkynes. Adv. Synth. Catal. 2010, 352, 1315-1337. (g) Crespo, O.; Gimeno, M. C.; Laguna, A.; Montanel-Pérez, S.; Villacampa, M. D. Facile Synthesis of Gold(III) Aryl-Carbene Metallacycles. Organometallics 2012, 31, 5520-5526. (h) Montanel-Pérez, S.; Herrera, R. P.; Laguna, A.; Villacampa, M. D.; Gimeno, M. C. The fluxional amine gold(III) complex as an excellent catalyst and precursor of biologically active acyclic carbenes. Dalton Trans. 2015, 44, 9052-9062.

(14) Calhorda, M. J.; Canales, F.; Gimeno, M. C.; Jiménez, J.; Jones, P. G.; Laguna, A.; Veiros, L. F. Gold(I)-Gold(III) Interactions in Polynuclear Sulfur-Centered Complexes. Synthesis and Structural Characterization of $\left[\mathrm{S}\left(\mathrm{Au}_{2} \mathrm{dppf}\right)\left\{\mathrm{Au}\left(\mathrm{C}_{6} \mathrm{~F}_{5}\right)_{3}\right\}\right]$ and $[\{\mathrm{S}$ $\left.\left.\left(\mathrm{Au}_{2} \mathrm{dppf}\right)\right\}_{2}\left\{\mathrm{Au}\left(\mathrm{C}_{6} \mathrm{~F}_{5}\right)_{2}\right\}\right]$ OTf (dppf = 1,1'-Bis(diphenylphosphino)ferrocene). Organometallics 1997, 16, 3837-3844.

(15) Usón, R.; Laguna, A.; Arrese, M. L. A New Approach to the Synthesis of Cationic, Neutral or Anionic Diarylgold(III) Complexes. Synth. React. Inorg. Met.-Org. Chem. 1984, 14, 557-567.

(16) Usón, R.; Laguna, A.; Laguna, M. (Tetrahydrothiophene)Gold(I) or Gold(III) Complexes. Inorg. Synth. 1989, 26, 85-91.

(17) Usón, R.; Laguna, A. Polyaryl Derivatives of Gold(I), Silver(I) and Gold(III). Organomet. Synth. 1986, 3, 322-342.

(18) Sheldrick, G. M. Crystal structure refinement with SHELXL. Acta Cryst 2015, 71, 3-8. 